OPEN ACCESS

Edited by:

Filippo Brighina,

University of Palermo, Italy

Reviewed by:

Katharine Dunlop,

University of Toronto, Canada

Daniel Keeser,

Ludwig Maximilian University

of Munich, Germany

*Correspondence:

Keiichiro Nishida

nishidak@takii.kmu.ac.jp

Received: 28 March 2019

Accepted: 16 July 2019

Published: 08 August 2019

Citation:

Nishida K, Koshikawa Y, Morishima $Y$, Yoshimura $M$ Katsura K, Ueda S, Ikeda S, Ishii R, Pascual-Marqui $R$ and Kinoshita $T$ (2019) Pre-stimulus Brain Activity Is

Associated With State-Anxiety

Changes During Single-Session

Transcranial Direct Current

Stimulation.

Front. Hum. Neurosci. 13:266. doi: 10.3389/fnhum.2019.00266

\section{Pre-stimulus Brain Activity Is Associated With State-Anxiety Changes During Single-Session Transcranial Direct Current Stimulation}

\author{
Keiichiro Nishida ${ }^{1 *}$, Yosuke Koshikawa ${ }^{1}$, Yosuke Morishima ${ }^{2}$, Masafumi Yoshimura1, \\ Koji Katsura', Satsuki Ueda', Shunichiro Ikeda', Ryouhei Ishii ${ }^{3}$, \\ Roberto Pascual-Marqui ${ }^{1,4}$ and Toshihiko Kinoshita ${ }^{1}$
}

${ }^{1}$ Department of Neuropsychiatry, Kansai Medical University, Osaka, Japan, ${ }^{2}$ Division of Systems Neuroscience of Psychopathology, Translational Research Center, University Hospital of Psychiatry, University of Bern, Bern, Switzerland, ${ }^{3}$ Osaka Prefecture University Graduate School of Comprehensive Rehabilitation, Osaka University, Osaka, Japan, ${ }^{4}$ The KEY Institute for Brain-Mind Research, University of Zurich, Zurich, Switzerland

Transcranial direct current stimulation is a promising neuromodulation method for treating depression. However, compared with pharmacological treatment, previous studies have reported that a relatively limited proportion of patients respond to tDCS treatment. In addition, the neurophysiological mechanisms underlying tDCS treatment remain unclear, making it difficult to identify response predictors for tDCS treatment based on neurophysiological function. Because treatment effects are achieved by repetitive application of tDCS, studying the immediate effects of tDCS in depressive patients could extend understanding of its treatment mechanisms. However, immediate changes in a single session of tDCS are not well documented. Thus, in the current study, we focused on the immediate impact of tDCS and its association with prestimulus brain activity. To address this question, we applied anodal tDCS to the left dorsolateral prefrontal cortex (DLPFC) or dorsomedial prefrontal cortex (DMPFC) in 14 patients with major depressive disorder (MDD) and 19 healthy controls (HCs), at an intensity of $1.0 \mathrm{~mA}$ for $20 \mathrm{~min}$ in a single session. To evaluate anxiety, the state trait anxiety inventory was completed before and after tDCS. We recorded resting electroencephalography before tDCS, and calculated electrical neuronal activity in the theta and alpha frequency bands using standardized low-resolution electromagnetic tomography. We found that, during application of left DLPFC tDCS to patients with MDD, the anxiety reduction effect of tDCS was related to higher baseline theta-band activity in the rostral anterior cingulate cortex ( $\mathrm{rACC}$ ) and no medication with benzodiazepine used as hypnotic. For DMPFC stimulation in MDD, the anxiety reduction effect was associated with lower baseline alpha-band activity in the left inferior parietal lobule. In contrast, in $\mathrm{HCs}$, the anxiety reduction effect was associated with higher baseline alpha activity in the precuneus during DMPFC stimulation. The current results suggest that the association between pre-tDCS brain activity and the anxiety reduction effect of 
tDCS depends on psychopathology (depressed or non-depressed) as well as the site of stimulation (DMPFC or left DLPFC) and insomnia. Furthermore, the results suggest that tDCS response might be associated with baseline resting state electrophysiological neural activity.

Keywords: transcranial direct current stimulation, left dorsolateral prefrontal cortex, dorsomedial prefrontal cortex, anterior cingulate, anxiety, depression

\section{INTRODUCTION}

Transcranial direct current stimulation is a widely used neuromodulation technique for basic neurocognitive research in healthy subjects as well as clinical applications in major depression and other psychiatric disorders (Fregni et al., 2015; Martin et al., 2018) In clinical practice, the development of new treatment approaches without medication is important for patients, who show low tolerance to pharmacotherapy because of substantial side effects (Brunoni et al., 2012). tDCS provides a potentially useful approach because the tDCS stimulator is a mechanically simple device, with a lower cost than other non-invasive brain stimulation devices.

In recent decades, major depressive disorder (MDD) has become one of the most serious lifetime diseases in many countries (Murray and Lopez, 1996). Although treatments for MDD have improved, current treatment options have limitations (Kupfer et al., 2012).

In treatment methods involving non-invasive brain stimulation for MDD, the left dorsolateral prefrontal cortex (DLPFC) has been found to play a major role in executive functioning, and is widely recognized as a suitable target for anodal tDCS to recover executive control and emotion regulation.

A recent meta-analysis supports the application of tDCS to the DLPFC in MDD (Mutz et al., 2018). Furthermore, a recent large-scale study reported no inferiority of tDCS treatment compared with escitalopram (Brunoni et al., 2017). However, the specific treatment effects of tDCS remain controversial (Tremblay et al., 2014; Mondino et al., 2015; Brunoni et al., 2017; Martin et al., 2018). A recent study by Brunoni and colleagues reported that response rates to tDCS were significantly higher than placebo, but the remission rate was not significantly different between tDCS and placebo groups (Brunoni et al., 2017). Furthermore, the treatment mechanisms of tDCS remain unclear.

Recently, several studies proposed additional targets for treatment of MDD, suggesting non-invasive brain stimulation of the dorsomedial prefrontal cortex (DMPFC) as one potential approach (Downar and Daskalakis, 2013). This proposal is based on the finding that the DMPFC, including the anterior cingulate cortex, is involved in regulation of emotions (Bush et al., 2000), and is anatomically connected with the amygdala and nucleus accumbens, which have both been implicated in MDD.

More recent studies have confirmed the feasibility of rTMS on the DMPFC for MDD (Downar et al., 2014; Kreuzer et al., 2015; Schulze et al., 2018). However, we know little evidence of the mechanism of DMPFC stimulation even beyond the context of major depression (Bakker et al., 2015; Colzato et al., 2015; Kreuzer et al., 2015).

There are several limitations of the current evidence supporting further implementation of tDCS into clinical practice. First, better understanding of the neurophysiological mechanisms underlying the effects of tDCS is needed. Second, biomarkers are needed for predicting tDCS treatment responders. One possible approach for addressing these current limitations is to examine the neurophysiological signatures of patients. Specifically, the pre-stimulus state of the brain may explain the variability in responses to tDCS. Recent studies have reported that pre-treatment electroencephalography (EEG) predicts changes in cognition after 15 sessions of tDCS in the left DLPFC in depressive patients, and that frontal electrodes exhibit predictive power for changes in cognition (Al-Kaysi et al., 2016, 2017).

Although predicting treatment effects with pre-treatment neurophysiological activity would have direct implications for clinical practice, the neurophysiological mechanisms underlying treatment effects may remain obscured because treatment effects are achieved by repeated application of single-session tDCS, and the accumulation of immediate neural responses to singlesession tDCS may modify the stable state of brain activity and eventually improve depressive symptoms. Therefore, we assumed that examining the neural mechanisms of a singlesession of tDCS intervention might provide a first step for disentangling the complex treatment mechanisms of tDCS for MDD. Among symptomatic problems of MDD, single session of tDCS is hard to change sustained symptoms such as depressive mood, anhedonia, agitation or loss of motivation, while anxiety is relatively volatile across time. In the current study, we therefore focus on state anxiety to look at the effect of singlesession tDCS.

In the current study, we set two main aims. First, we investigated the immediate effects of prefrontal tDCS on brain activity and state anxiety. Second, we compared the effects between stimulation of the left DLPFC, the canonical target of tDCS for MDD, and the DMFPC, the potential target predicted by neuroanatomical architecture. To this end, we applied anodal tDCS to the left DLPFC or DMPFC in MDD patients and healthy controls (HCs), with 20 min of stimulation in a single session. We measured state anxiety before and after tDCS and neural activity with EEG before tDCS. Finally, we examined association between neural activity and state anxiety to investigate neural predictors of the change in anxiety induced by tDCS. 


\section{MATERIALS AND METHODS}

\section{Participants}

We recorded a total of 20 patients with MDD, assessed by the DSM-IV and evaluated with the Hamilton Depression Rating Scale (HAM-D), and a total $24 \mathrm{HCs}$ subjects recruited for this study. After eliminating data corresponding to subjects that were left-handed, or unavailable EEG, or psychological evaluation, 14 patients with MDD and $19 \mathrm{HCs}$ were finally included in this study. All participants were right-handed, and were graduates of high school or higher education. All participants were diagnosed by experienced psychiatrists using a structured interview and physical examination. We excluded patients with history of dementia, schizophrenia, substance dependence, epilepsy or head trauma. Participants do not have anxiety disorder comorbidities, such as generalized anxiety disorder, panic disorder, and phobia. Thirteen patients have received antidepressant. 10 patients were medicated by benzodiazepine as sleeping medication and 2 patients medicated by a mood stabilizer. Chi-squared test with Yates's correction between gender did not show significant difference $\left(x^{2}(1)=3.076, p(0.08)\right.$. All HCs had no history of psychiatry disorders. This study was carried out in accordance with the recommendations of "Safety of transcranial direct current stimulation, tDCS by Japanese Journal of Clinical Neurophysiology 2011.”

The study protocol was approved by the Institutional Ethics Review Committee of Kansai Medical University (UMIN000015046). We obtained written informed consent from all participants in accordance with the Declaration of Helsinki. Participants were recruited from September 2014 to April 2017. Details of participants are shown in Table 1.

\section{tDCS}

tDCS was administered with a battery-driven stimulator (DC Stimulator Plus, Neuroconn, Ilmenau, Germany). The electrical current was applied at $1 \mathrm{~mA}$ via electrically conductive rubber electrodes $\left(20 \mathrm{~cm}^{2}\right.$, circular in shape) attached with an adhesive conductive EEG paste. Anodal stimulation was administered over the left-DLPFC (F5, 1010 EEG international electrode placement, Figure 1) or the DMPFC (AFz, 10-10 EEG international electrode placement, Figure 2) with the cathodal electrode placed on the left shoulder. Direct current was administered for $20 \mathrm{~min}$ during the resting state. We also simulated the current flow of our montage with a simulation software using a finite element model (HD-explore, Soterix Medical, New York, United States) (Figures 1, 2).

\section{Procedure}

We adopted a between-subjects cross-over design (Figure 3). The order of stimulation was counterbalanced. Each subject was randomly assigned to receive left DLPFC or DMPFC tDCS in the first session. The participant received tDCS on the other site in the second session. There was an interval of at least 1 week between $\mathrm{tDCS}$ sessions.
TABLE 1 | Demographic data.

\begin{tabular}{|c|c|c|c|c|}
\hline \multirow{2}{*}{$\begin{array}{l}\text { Group } \\
\text { Session }\end{array}$} & \multicolumn{2}{|c|}{ MDD } & \multicolumn{2}{|c|}{$\mathrm{HC}$} \\
\hline & Left DLPFC & DMPFC & Left DLPFC & DMPFC \\
\hline Sample size & \multicolumn{2}{|c|}{14} & \multicolumn{2}{|c|}{19} \\
\hline $\begin{array}{l}\text { Sex: } \\
\text { Male/female }\end{array}$ & \multicolumn{2}{|c|}{$12 / 2$} & \multicolumn{2}{|c|}{$12 / 7$} \\
\hline \multicolumn{5}{|l|}{ Drug treatment } \\
\hline No & \multicolumn{2}{|c|}{1} & \multicolumn{2}{|c|}{0} \\
\hline $\begin{array}{l}\text { One } \\
\text { antidepressant }\end{array}$ & \multicolumn{2}{|c|}{11} & \multicolumn{2}{|c|}{0} \\
\hline $\begin{array}{l}\text { Two } \\
\text { antidepressants }\end{array}$ & \multicolumn{2}{|c|}{2} & \multicolumn{2}{|c|}{0} \\
\hline Benzodiazepine & \multicolumn{2}{|c|}{10} & \multicolumn{2}{|c|}{0} \\
\hline Mood stabilizer & \multicolumn{2}{|c|}{2} & \multicolumn{2}{|c|}{0} \\
\hline $\begin{array}{l}\text { Age-years: } \\
\text { mean } \pm S D\end{array}$ & \multicolumn{2}{|c|}{$44.93 \pm 14.68$} & \multicolumn{2}{|c|}{$48.94 \pm 15.80$} \\
\hline $\begin{array}{l}\text { Education } \\
\text { period }\end{array}$ & \multicolumn{2}{|c|}{$15.36 \pm 1.55$} & \multicolumn{2}{|c|}{$15.63 \pm 1.34$} \\
\hline $\begin{array}{l}\text { Number of } \\
\text { previous } \\
\text { episodes: } \\
\text { mean } \pm \text { SD }\end{array}$ & \multicolumn{2}{|c|}{$2.36 \pm 0.93$} & \multicolumn{2}{|c|}{$0.00 \pm 0.00$} \\
\hline $\begin{array}{l}\text { HAM-D17 } \\
\text { score on the } \\
\text { day of the } \\
\text { session: } \\
\text { mean } \pm \text { SD }\end{array}$ & $14.07 \pm 5.40$ & $13.79 \pm 4.82$ & $0.21 \pm 0.42$ & $0.21 \pm 0.54$ \\
\hline
\end{tabular}

A

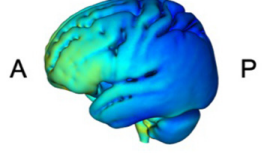

C

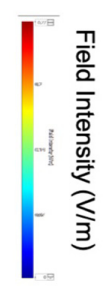

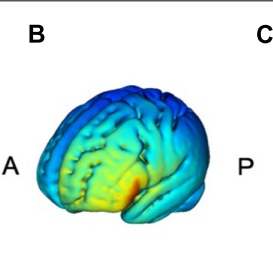

FIGURE 1 | Modeling of electric field distribution for the montage of left DLPFC stimulation. (A) Sagittal view, (B) side view, (C) above view, A: anterior, P: posterior.

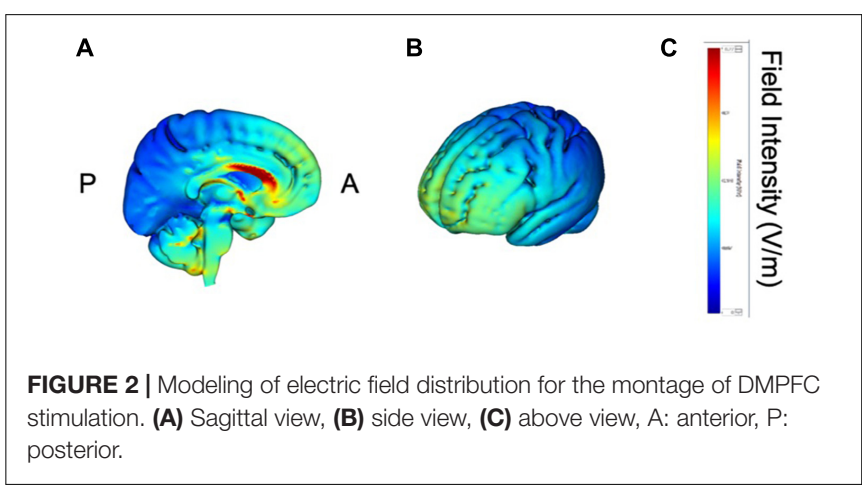

\section{Psychological Test}

We measured the STAI (state-trait anxiety inventory), which consists of two subscales, STAI-SA for state anxiety to 


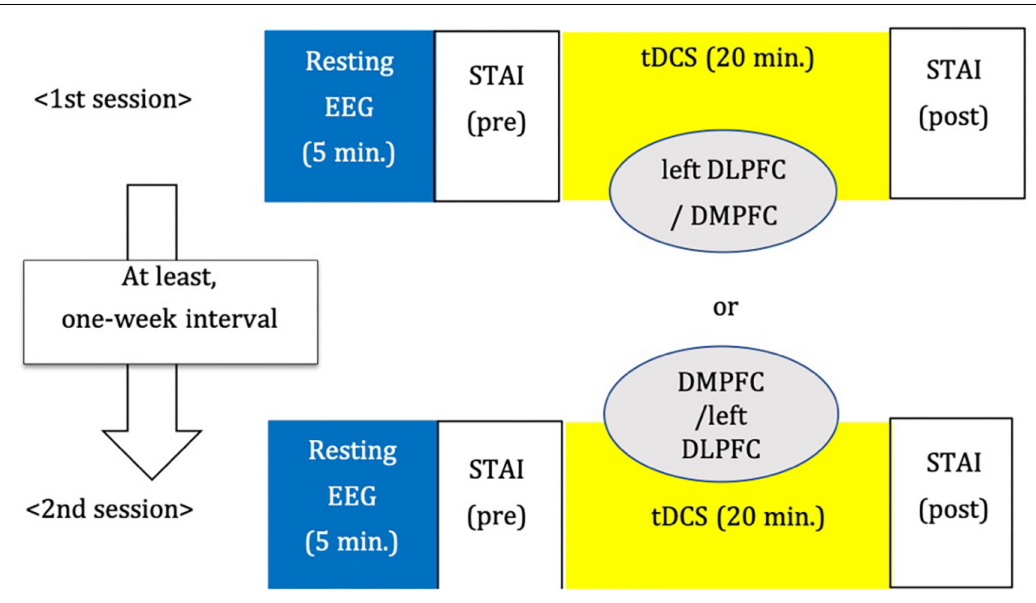

FIGURE 3 | Study design.

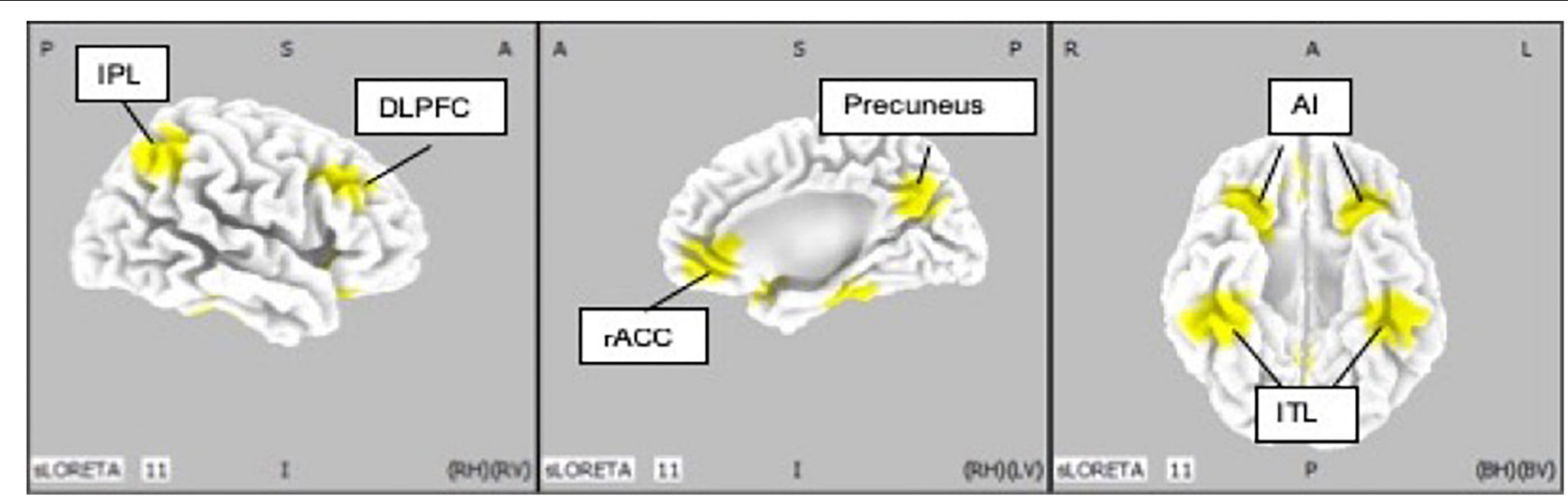

FIGURE 4 | Location of regions of interest (IP: inferior parietal lobe; DLPFC: dorsolateral prefrontal cortex; rACC: rostral anterior cingulate cortex; Al: anterior insula; ITL: inferior temporal lobe).

assess anxiety before and after tDCS and STAI-TA for trait anxiety before tDCS.

\section{EEG Recording}

Resting and eyes-closed EEG was recorded with an EEG-1200 Nihon Kohden (Tokyo, Japan) system. A $64 \mathrm{ch} \mathrm{Ag} / \mathrm{AgCl}$ sintered Waveguard Original EEG cap from ANT Neuro (Netherland) was used for the recordings. It was necessary to use a subset of the electrodes comprising of 19 EEG electrodes corresponding to the international 10-20 system for analyses, because the tDCS electrodes placed under the EEG cap interfered with substantial number of EEG electrodes in the frontal area. We recorded EEG before and after tDCS. However, only EEG recordings before tDCS were used in the analyses described below.

\section{EEG Analysis}

Signals of cortical electric neuronal activity were computed from the baseline, pre-stimulation EEG recordings using standardized low resolution electromagnetic tomography (sLORETA) (Pascual-Marqui, 2002). In its current implementation (free academic software package available at https://www.uzh.ch/keyinst/loreta), this method produces signals of appropriately standardized current density from 6239

TABLE 2 | Regions of interest (ROIs) and their coordinates in the MNI space.

\begin{tabular}{lrcc}
\hline & $\boldsymbol{x}$ & $\boldsymbol{y}$ & $\boldsymbol{z}$ \\
\hline rACC & 0 & 45 & 0 \\
Left DLPFC & -40 & 26 & 34 \\
Right DLPFC & 40 & 26 & 34 \\
Left Al & -30 & 24 & -13.5 \\
Right Al & 30 & 24 & -13.5 \\
Left ITL & -42 & -33 & -25.5 \\
Right ITL & 42 & -33 & -25.5 \\
Left IPL & -45 & -52 & 48 \\
Right IPL & 45 & -52 & 48 \\
Precuneus & 0 & -66 & 34
\end{tabular}

rACC, rostral anterior cingulate cortex; DLPFC, dorsolateral prefrontal cortex; Al, anterior insula; ITL, inferior temporal lobe; IPL, inferior parietal lobe. 
TABLE 3 | STAI-SA pre tDCS and STAI-SA post tDCS

\begin{tabular}{lccccc}
\hline Group & \multicolumn{2}{c}{ MDD } & & \multicolumn{2}{c}{ HC } \\
\cline { 2 - 3 } \cline { 5 - 6 } Session & Left DLPFC & DMPFC & & Left DLPFC & DMPFC \\
\hline $\begin{array}{l}\text { STAl- } \\
\text { SA_pre }\end{array}$ & $47.29( \pm 9.10)$ & $44.71( \pm 8.11)$ & & $36.32( \pm 7.02)$ & $35.84( \pm 6.80)$ \\
$\begin{array}{l}\text { STAl- } \\
\text { SA_post }\end{array}$ & $44.64( \pm 9.96)$ & $43.14( \pm 9.81)$ & & $38.42( \pm 6.40)$ & $37.16( \pm 6.52)$ \\
$\begin{array}{l}\text { Post-Pre } \\
\text { change of } \\
\text { STAI-SA }\end{array}$ & $-2.64( \pm 5.23)$ & $-1.57( \pm 6.01)$ & & $2.11( \pm 5.86)$ & $1.32( \pm 4.35)$ \\
\hline
\end{tabular}

DLPFC, dorsolateral prefrontal cortex stimulation; DMPFC: dorsomedial prefrontal cortex stimulation, MDD: major depressive disorder, HC: healthy controls, STAI-SA: State-Trait Anxiety Inventory - state anxiety.

cortical gray matter voxels, sampled on a $5 \mathrm{~mm}$ resolution grid, using the MNI152 anatomical template (Mazziotta et al., 2001; Fuchs et al., 2002). sLORETA has received both theoretical
(Greenblatt et al., 2005; Sekihara et al., 2005; Pascual-Marqui, 2007) and experimental validation (Pascual-Marqui et al., 2009).

The sLORETA signals were then further processed to produce values of cortical spectral power in two classical EEG frequency bands: theta $(4-8 \mathrm{~Hz})$ and alpha $(8-12 \mathrm{~Hz})$. We chose these two frequency bands because they have been repeatedly reported to be associated with MDD and response to treatment (Klimesch, 1999; Moore et al., 2000; Nishida et al., 2015; Kitaura et al., 2017).

\section{Regions of Interest}

Ten regions of interest (ROIs) were chosen based on previous studies investigating neurophysiological mechanisms in patients with MDD (McGrath et al., 2013; Kaiser et al., 2015; Pizzagalli et al., 2018) (Figure 4). Pizzagalli et al., investigated the importance of current density in rACC for improvement of depression symptoms with EEG-LORETA. The meta-analysis by Kaiser et al., showed significant difference in resting state

TABLE 4 | Single regression analysis about STAI-SA.

\begin{tabular}{|c|c|c|c|c|c|c|c|c|}
\hline Session & Dependent variable & Independent variable & $\beta$ & SE $\beta$ & $t$-value & $p$-value & Standard $\beta$ & $R 2$ \\
\hline MDD & Change of STAI-SA & STAI-SA pre & -0.064 & 0.127 & -0.507 & 0.616 & -0.099 & -0.028 \\
\hline $\mathrm{HC}$ & Change of STAI-SA & STAI-SA pre & -0.339 & 0.111 & -3.047 & 0.004 & $-0.453^{* *}$ & 0.183 \\
\hline
\end{tabular}

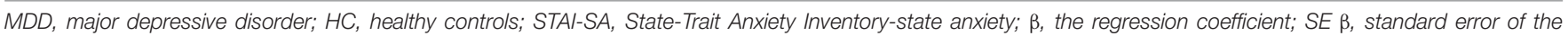
regression coefficient; $R^{2}$, the squared multiple correlation coefficient.

TABLE 5 | Log-transformed current density power at 10 ROls in alpha and theta bands.

\begin{tabular}{|c|c|c|c|c|c|c|c|c|}
\hline $\begin{array}{l}\text { Group } \\
\text { Session } \\
\text { Current density }\end{array}$ & \multicolumn{4}{|c|}{ MDD } & \multicolumn{4}{|c|}{ HC } \\
\hline Theta_rACC & 0.186 & 0.518 & 0.133 & 0.570 & 0.521 & 0.519 & 0.400 & 0.454 \\
\hline Theta_leftDLPFC & -0.380 & 0.459 & -0.386 & 0.411 & -0.079 & 0.526 & -0.153 & 0.417 \\
\hline Theta_rightInsula & 0.138 & 0.585 & 0.215 & 0.531 & 0.276 & 0.516 & 0.279 & 0.447 \\
\hline Theta_IrftITP & -0.350 & 0.413 & -0.246 & 0.247 & -0.003 & 0.437 & -0.084 & 0.366 \\
\hline Theta_rightITP & -0.328 & 0.467 & -0.199 & 0.376 & -0.115 & 0.438 & -0.264 & 0.466 \\
\hline Theta_leftIPL & -0.531 & 0.481 & -0.667 & 0.378 & -0.216 & 0.426 & -0.119 & 0.618 \\
\hline Theta_rightIPL & -0.543 & 0.486 & -0.637 & 0.433 & -0.253 & 0.481 & -0.254 & 0.606 \\
\hline Alpha_leftlnsula & -0.056 & 0.545 & 0.009 & 0.230 & 0.157 & 0.273 & 0.039 & 0.292 \\
\hline Alpha_rightInsula & 0.116 & 0.590 & 0.185 & 0.376 & -0.028 & 0.311 & -0.099 & 0.257 \\
\hline Alpha_Irft|TP & 0.233 & 0.794 & 0.535 & 0.494 & 0.381 & 0.614 & 0.299 & 0.592 \\
\hline Alpha_rightITP & 0.404 & 0.925 & 0.621 & 0.633 & 0.316 & 0.616 & 0.064 & 0.725 \\
\hline Alpha_leftIPL & -0.010 & 0.793 & 0.290 & 0.705 & 0.140 & 0.706 & 0.327 & 0.763 \\
\hline Alpha_rightIPL & 0.173 & 0.857 & 0.460 & 0.706 & 0.210 & 0.612 & 0.179 & 0.793 \\
\hline Alpha_precuneus & 0.254 & 0.881 & 0.483 & 0.758 & 0.281 & 0.710 & 0.357 & 0.826 \\
\hline
\end{tabular}

DLPFC, dorsolateral prefrontal cortex; DMPFC, dorsomedial prefrontal cortex, rACC, rostral anterior cingulate cortex; Al, anterior insula; ITL, inferior temporal lobe; IPL, inferior parietal lobe; $M D D$, major depressive disorder; $H C$, healthy controls. 
functional connectivity in patients with depression and HCs. In addition, McGrath et al. have shown that anterior insula and inferior temporal lobe were candidates of biomarkers of treatment by cognitive behavior therapy by using positron emission topography.

We defined rACC from average coordinates (i.e., centroid) of the atlas which Pizaggali et al. used in their paper. Atlas of DLPFC and Inferior temporal lobe in our study was obtained from Kaiser's literature, and the coordinates used for the anterior insula and inferior temporal lobe originate from the work of McGrath (McGrath et al., 2013; Table 2).

\section{Statistical Analysis}

For each stimulation session, the change in anxiety scores was defined as the STAI-SA score at post tDCS minus the STAI-SA score at pre tDCS (baseline). Thus, a negative value of the change indicates a reduction of state anxiety. In the current study, we aimed to investigate the association between these dependent variables and cortical activities in 10 ROIs. We also included the "with" or "without" administration of benzodiazepines as an independent variable, and baseline STAI-SA scores for considering the effect of the diversity of participants.

Firstly, we applied a least absolute shrinkage and selection operator (LASSO) for selecting appropriated variables and regularization. The set of independent variables consisted of the cortical spectral power for the theta and alpha bands, at 10 ROIs calculated from baseline, pre-stimulation EEG-sLORETA for each DLPFC or DMPFC session, plus the medication about with/without benzodiazepines and STAI-SA scores at pre-tDCS in patients and controls separately. Cross-Validation leave-oneout was performed to determine the optimal tuning penalty parameter $(\lambda)$ for each session. Finally, variable selection was performed by using the estimated $\lambda$ value. We performed LASSO with R (3.6.0), RStudio (1.2.1335), and glmnet package (2.0-18).

Next, forced entry multiple regression analyses were conducted for changes in STAI-SA scores as dependent variables, with the set of cortical activity in each theta and alpha band at selected independent variable, for both left DLPFC and DMPFC stimulation session, in the MDD group and in the control group. SPSS version 26 was used for this multiple regression analysis.

\section{Adverse Events}

Six of 23 participants reported headaches, tingling sensation, itching, or experiencing the taste of iron. Because all reported events were mild, all participants continued the experiments and recovered from the adverse effects immediately after the sessions.

\section{RESULTS}

\section{Change in STAI Scores}

We first examined overall changes in state anxiety. Table 3 shows the baseline and the Post-Pre tDCS changes in STAI-SA scores (Table 3).

Analysis of variance revealed that the baseline STAI-SA score was significantly higher in the MDD group than that in the HC group $(F[3,62]=8.943, p<0.001)$. However, analysis of covariance revealed no significant difference in changes of STAISA score between the two groups $(F[1,63]=1.562, p=0.215)$, and within each group (MDD: $F[1,25]=0.180, p=0.675$; HC: $F[1,35]=0.397, p=0.533)$. The paired $t$-test did not show the significantly between the score of pre-tDCS and the one of posttDCS in both MDD (left DLPFC session: $t=1.67, d f=14$, $p=0.12$, DMPFC session: $t=0.83, d f=14, p=0.42)$ and HC (left DLPFC session: $t=-1.57, d f=18, p=0.14$, DMPFC session: $t=-1.32, d f=18, p=0.20)$ groups.

In order to examine the influence of baseline STAI-SA score on tDCS-induced changes of STAI-SA, we performed single regression analysis where the independent variable is the baseline STAI-SA score for each group, and the dependent variable is as STAI-SA change (Table 4). We did not find significant association between pre-tDCS STAI-SA score and tDCS-induced changes of STAI-SA score in the MDD group $(p=0.61)$, while it was significant in the HC group $(\beta=-0.339$, $p=0.004)$. We also examined whether pre tDCS STAISA score was different between the subjects treated with benzodiazepine and those without benzodiazepine. MannWhitney $U$-tests did not show the significant difference between the two groups $(p=0.72)$.

TABLE 6 | Least absolute shrinkage and selection operator (LASSO) for selecting appropriated variables and regularization.

\begin{tabular}{|c|c|c|c|c|}
\hline Sessions & $\begin{array}{l}\text { Dependent } \\
\text { variable }\end{array}$ & Independent variable & $\lambda$ & $\beta$ \\
\hline \multirow[t]{2}{*}{$\begin{array}{l}\text { MDD on left } \\
\text { DLPFC }\end{array}$} & $\begin{array}{l}\text { Change of } \\
\text { STAI-SA }\end{array}$ & Theta rACC & 0.199 & -0.375 \\
\hline & & Benzodiazepine & & -0.752 \\
\hline \multirow[t]{3}{*}{$\begin{array}{l}\text { MDD on left } \\
\text { DLPFC }\end{array}$} & $\begin{array}{l}\text { Change of } \\
\text { STAI-SA }\end{array}$ & Alpha left ITL & 0.227 & 0.285 \\
\hline & & Alpha precuneus & & 0.187 \\
\hline & & Benzodiazepine & & -0.414 \\
\hline \multirow[t]{3}{*}{$\begin{array}{l}\text { MDD on } \\
\text { DMPFC }\end{array}$} & $\begin{array}{l}\text { Change of } \\
\text { STAI-SA }\end{array}$ & Theta left DLPFC & 0.262 & -0.199 \\
\hline & & Theta right insula & & -0.036 \\
\hline & & Theta precuneus & & 0.205 \\
\hline \multirow[t]{4}{*}{$\begin{array}{l}\text { MDD on } \\
\text { DMPFC }\end{array}$} & $\begin{array}{l}\text { Change of } \\
\text { STAI-SA }\end{array}$ & Alpha rACC & 0.156 & -0.018 \\
\hline & & Alpha left DLPFC & & -0.184 \\
\hline & & Alpha right ITL & & 0.114 \\
\hline & & Alpha left IPL & & 0.544 \\
\hline $\begin{array}{l}\mathrm{HC} \text { on left } \\
\text { DLPFC }\end{array}$ & $\begin{array}{l}\text { Change of } \\
\text { STAI-SA }\end{array}$ & Theta n.s. & 0.503 & n.s. \\
\hline $\begin{array}{l}\text { HC on left } \\
\text { DLPFC }\end{array}$ & $\begin{array}{l}\text { Change of } \\
\text { STAI-SA }\end{array}$ & Alpha n.s. & 0.503 & n.s. \\
\hline $\begin{array}{l}\text { HC on } \\
\text { DMPFC }\end{array}$ & $\begin{array}{l}\text { Change of } \\
\text { STAI-SA }\end{array}$ & Theta n.s. & 0.244 & n.s. \\
\hline \multirow[t]{2}{*}{$\begin{array}{l}\mathrm{HC} \text { on } \\
\text { DMPFC }\end{array}$} & $\begin{array}{l}\text { Change of } \\
\text { STAI-SA }\end{array}$ & Alpha rACC & 0.220 & 0.129 \\
\hline & & Alpha precuneus & & -0.403 \\
\hline
\end{tabular}

rACC, rostral anterior cingulate cortex; DLPFC, dorsolateral prefrontal cortex; ITL, inferior temporal lobe; IPL, inferior parietal lobe; DLPFC, dorsolateral prefrontal cortex stimulation; DMPFC stimulation, dorsomedial prefrontal cortex; MDD, major depressive disorder; $\mathrm{HC}$, healthy controls; $\beta$, the regression coefficient; $\lambda$, tuning penalty parameter; n.s, no significance. 
TABLE 7 | Regression analysis in each theta and alpha bands at on left DLPFC and MDD in patients and HC.

\begin{tabular}{|c|c|c|c|c|c|c|c|c|}
\hline Session & Dependent variable & Independent variable & $\beta$ & SE $\beta$ & $t$-value & $p$-value & Standard $\beta$ & $\boldsymbol{R}$ \\
\hline \multirow[t]{2}{*}{ MDD on left DLPFC } & Change of STAI-SA & Theta rACC & -5.912 & 1.809 & -3.268 & $0.007^{* *}$ & -0.586 & 0.583 \\
\hline & & benzodiazepine & -6.281 & 1.998 & -3.144 & $0.009 * *$ & -0.563 & \\
\hline \multirow[t]{3}{*}{ MDD on left DLPFC } & Change of STAI-SA & Alpha left ITL & 2.571 & 1.915 & 1.343 & 0.209 & 0.390 & 0.598 \\
\hline & & Alpha Precuneus & 1.728 & 1.725 & 1.002 & 0.340 & 0.291 & \\
\hline & & benzodiazepine & -4.181 & 2.039 & -2.051 & 0.067 & -0.375 & \\
\hline \multirow[t]{3}{*}{ MDD on DMPFC } & Change of STAI-SA & Theta left DLPFC & -6.600 & 6.328 & -1.043 & 0.321 & -0.451 & 0.327 \\
\hline & & Theta right Insula & -0.428 & 5.022 & -0.085 & 0.934 & -0.038 & \\
\hline & & Theta Precuneus & 5.299 & 2.768 & 1.914 & 0.085 & 0.460 & \\
\hline \multirow[t]{4}{*}{ MDD on DMPFC } & Change of STAI-SA & Alpha rACC & -2.237 & 3.497 & -0.640 & 0.538 & -0.123 & 0.679 \\
\hline & & Alpha left DLPFC & -6.678 & 4.630 & -1.442 & 0.183 & -0.268 & \\
\hline & & Alpha right ITL & 2.259 & 1.735 & 1.302 & 0.225 & 0.238 & \\
\hline & & Alpha left IPL & 5.210 & 1.587 & 3.283 & $0.009 * *$ & 0.611 & \\
\hline HC on left DLPFC & Change of STAI-SA & Theta n.s. & - & - & - & - & - & - \\
\hline HC on left DLPFC & Change of STAI-SA & Alpha n.s. & - & - & - & - & - & - \\
\hline HC on DMPFC & Change of STAI-SA & Theta n.s. & - & - & - & - & - & - \\
\hline \multirow[t]{2}{*}{$\mathrm{HC}$ on DMPFC } & Change of STAI-SA & Alpha rACC & 3.416 & 2.349 & 1.454 & 0.165 & 0.282 & 0.481 \\
\hline & & Alpha precuneus & -2.923 & 1.019 & -2.869 & $0.011^{*}$ & -0.556 & \\
\hline
\end{tabular}

rACC, rostral anterior cingulate cortex; DLPFC, dorsolateral prefrontal cortex; ITL, inferior temporal lobe; IPL, inferior parietal lobe; MDD, major depressive disorder, HC; healthy controls. ${ }^{*} p<0.05,{ }^{* *} p<0.01$.

\section{Patients with Depression}
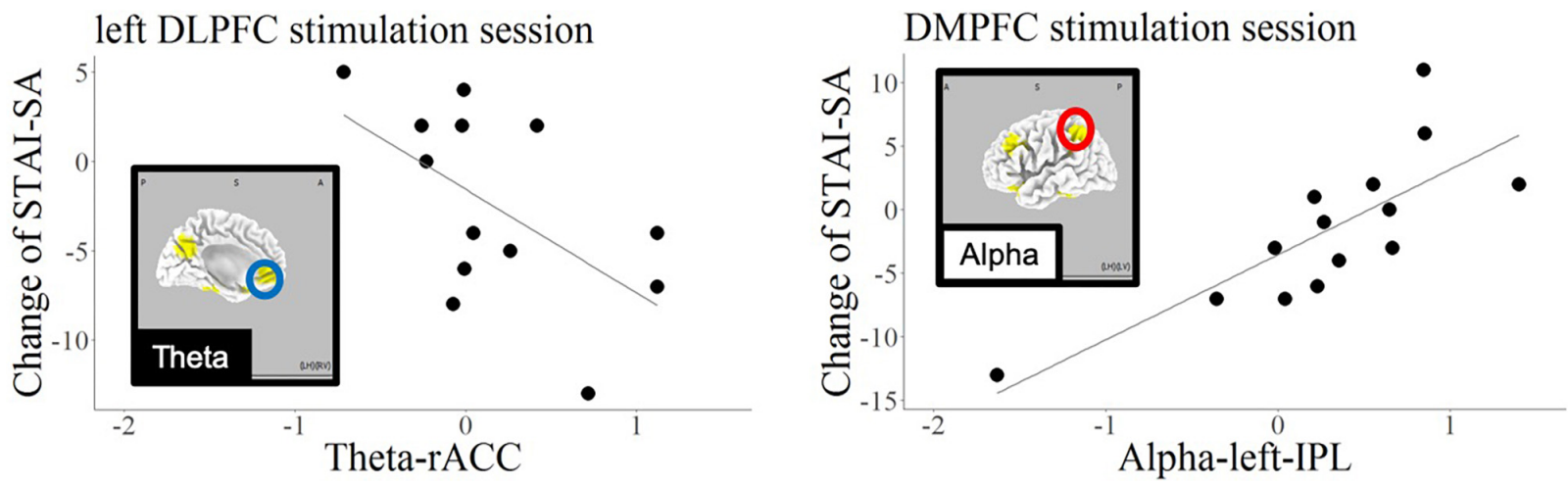

FIGURE 5 | Schematic summary of results in patients with MDD, based on Table 7. The red circles enclose regions with a positive slope (B > 0), and best responders with negative STAl-change corresponded to decreased cortical activity. The blue circle encloses a region with a negative slope $(B<0)$, and best responders with negative STAI-change corresponded to increased cortical activity.

\section{Brain Activity in ROls}

We further examined pre-tDCS brain activity estimated by sLORETA. Table 5 shows the values of brain activity calculated by LORETA. Paired $t$-tests revealed no significant difference in log-transformed current density power between the DLPFC and DMPFC sessions, in each of the MDD and HC groups (MDD: $p=0.299$; HC: $p=0.255$ ).

\section{Multiple Linear Regression Models}

To examine whether pre-tDCS brain activity can be associated with anxiolytic effect, we first performed Lasso regression to select predictor variables of each set of theta or alpha activity
(Table 6). Here we also included benzodiazepine medication as a predictor variable to control effect of benzodiazepine medication. We then further performed multiple regression analysis if selected variables was associated with tDCSinduced STAI-SA changes. Table 7 shows the results of forced entry multiple linear regression models. Negative values for STAI change corresponded to a reduction of state anxiety after tDCS.

Figures 5, 6 show the schematic summaries of the MDD and HC group with significantly difference $(P<0.05)$. We modeled the change in STAI-SA scores in vertical axis, and the set of cortical theta and alpha activity in 10 ROIs in horizonal axis for left DLPFC and DMPFC stimulation, and in each group. 


\section{Healthy controls}

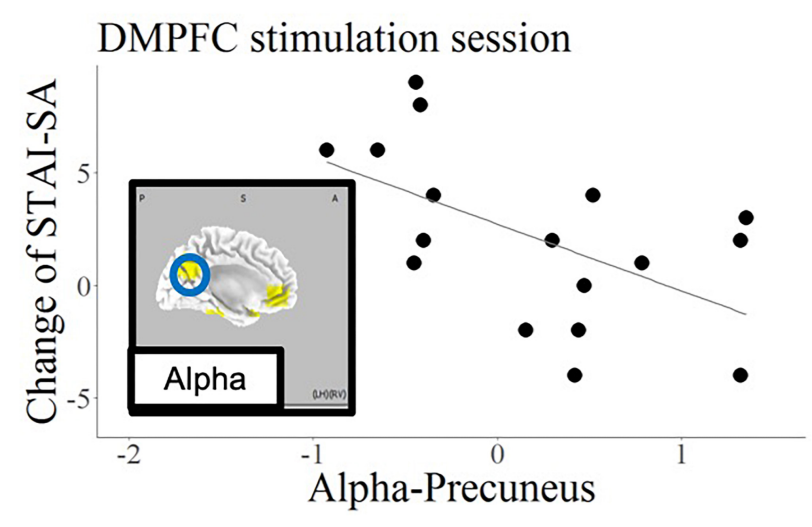

FIGURE 6 | Schematic summary of results in the HC group, based on Table 7. The blue circle encloses a region with a negative slope $(B<0)$, and best responders with negative STAI-change corresponded to increased cortical activity.

\section{DISCUSSION}

The current findings confirmed that, regarding the left DLPFC stimulation site in patients with MDD, the anxiety reduction effect of tDCS was related to higher baseline theta-band activity in the rostral anterior cingulate cortex (rACC). In contrast, the anxiety reduction was associated with higher baseline alpha activity in the precuneus in the HC group.

In the current study, we have specifically focused on immediate anxiolytic effect of tDCS, and we did not expect to change sustained depressive symptoms. We indeed consider accumulation of immediate anxiolytic effects will eventually lead to long-term improvement of depressive symptoms.

The association of the anxiolytic effect of left DLPFC tDCS with high baseline theta-band activity in the rACC is in accord with the findings of previous studies (Arns et al., 2014; Li et al., 2014; Bailey et al., 2018). A large scale meta-analysis studies have further shown that functional and structural alterations in the rACC are associated with broad spectrum of psychiatric disorders (Goodkind et al., 2015; Sha et al., 2019). Patients with MDD have been reported to exhibit dysfunction in the left DLPFC as well as the rACC (Pizzagalli et al., 2002; Mayberg et al., 2005). A study for treatment resistant depression patients who were administered with rTMS for 4 to 7 weeks showed the antidepressive effect was predicted by functional connectivity between stimulation site and the subgenual cingulate (Weigand et al., 2018). Liston and colleagues also reported that activation of the subcallosal cingulate cortex was a main predictor for the effect of transcranial magnetic stimulation (Liston et al., 2014). Pizzagalli and colleagues reported that LORETA current density of theta-band in the rACC was a predictor of response to antidepressants. The current findings are in accord with these previous studies, and further suggest that the rACC, including the subcallosal cingulate cortex, is involved in the anxiolytic effects of
tDCS applied to the left DLPFC, and may appear to be important for predicting the response of MDD patients to tDCS.

The current findings also revealed a correlation between baseline alpha-band activity in the IPL and state anxiety reduction during DMPFC stimulation in the MDD patient group. Several previous studies have examined the relationship between anxiety and functional brain imaging in IPL (Hasler et al., 2007; Goldin et al., 2009). Importantly, the current results revealed opposite prediction patterns in patients with MDD and HCs; the best responding HCs (exhibiting negative STAI change) were those with high alpha activity in precuneus in response to DMPFC stimulation.

A study by Fox et al. suggested that the region for stimulation by neuromodulation can be selected not only by the direct effect of the stimulation, but also by the propagation effect, depending on the interconnected regions of the resting state networks (Fox et al., 2014).

As the precuneus and ACC constitute the default mode network (Sheline et al., 2009), applying tDCS to the DMPFC might affect activity in the precuneus, which is functionally densely connected with the DMPFC. This association was only found in the $\mathrm{HC}$ group, presumably reflecting intact functionality of the default mode network in healthy individuals. Importantly, other remote effects of tDCS have been reported in previous studies. tDCS applied to the left DLPFC was reported to increase functional connectivity in the fronto-parietal network, while decreasing connectivity in the default mode network (van der Werf et al., 2010; Eldaief et al., 2011). It should be noted that anxiety disorder and depression are likely to be related to dysfunction of this frontal-parietal network (Sylvester et al., 2012). And this dysfunction also may yield the opposite changing of STAI-SA; decreasing the mean score of STAI-SA in MDD and increasing STAI-SA mean score in HCs.

Regarding the prediction of responses to tDCS treatment in patients with MDD, Al-Kay et al. conducted a prediction analysis with EEG data for treatment outcomes in response to prefrontal tDCS. The results revealed that frontal EEG channels were important for predicting mood improvement after treatment sessions (Al-Kaysi et al., 2017). In contrast to previous studies, the current study involved a single tDCS application, and did not examine predictors of overall treatment, but immediate responses to a single session of treatment. We believe determining the immediate neurophysiological effects of tDCS is particularly important for understanding the treatment mechanisms of tDCS, because the accumulation of immediate changes may eventually lead to long-term plasticity underlying the overall treatment effects. It should also be emphasized that sLORETA can localize activity in deeper subcortical regions, whereas scalp EEG electrodes provide limited information about the underlying cortical activity due to cortical surface orientation and volume conduction effects.

Interestingly, the patients taking no benzodiazepine medication had apparently an anxiety reduction effect for left DLPFC stimulation; however, there was no significantly difference pre-STAI scores between patients with and without benzodiazepine. The results is indeed consistent with a previous clinical trial. The clinical trial comparing the treatment response 
between tDCS and sertraline showed that benzodiazepine decreases the effect only in the tDCS treatment group (Brunoni et al., 2013). Benzodiazepine is used as hypnotics in this study, thus, it might be interesting to investigate interactions between tDCS and benzodiazepine relating to insomnia in future research.

Additionally, previous studies have reported slow EEG power changes before and after tDCS under the tasks, which would support further studies using slow frequencies (Keeser et al., 2011; Wirth et al., 2011; Jacobson et al., 2012; Ulam et al., 2015).

\section{LIMITATIONS}

Our current results, demonstrating the anxiety-reducing effects of tDCS in patients with MDD, will be of interest to researchers and clinicians who seek to use neuromodulation techniques as a novel treatment for depression. However, several limitations of the current study should be noted. First, the relatively small number of participants and no placebo stimulations may warrant some caution in the interpretation of these results. Second, because we applied only one stimulation, we did not examine the therapeutic effects of tDCS on depressive symptoms. Third, chi-squared test for gender imbalance between the MDD and $\mathrm{HC}$ groups tended to be imbalanced, suggesting gender effect may account for the difference between MDD and HC. However, this study design also has several strengths. First, we adopted with-in subject cross over design. Second, by adopting singlesession tDCS, we were able to reduce the total experiment time and burden on the subjects compared with experiments involving multiple stimulation sessions. In future research, a randomized controlled trial of tDCS intervention with a large number of participants would be helpful for addressing these limitations.

\section{CONCLUSION}

The current results revealed that the immediate anxiolytic effect of left DLPFC tDCS was associated with activity in the rACC and the left IPL, whereas DMPFC stimulation was correlated with activity in the precuneus. These findings suggest that the effects of tDCS are not only directly related to the stimulation area, but also to other brain areas involved in the same resting state networks. Further, we propose that pre-stimulus

\section{REFERENCES}

Al-Kaysi, A. M., Al-Ani, A., Loo, C. K., Breakspear, M., and Boonstra, T. W. (2016). "Predicting brain stimulation treatment outcomes of depressed patients through the classification of EEG oscillations," in Proceedings of the 2016 IEEE 38th Annual International Conference of the: IEEE Engineering in Medicine and Biology Society (EMBC), (Orlando, FL: IEEE).

Al-Kaysi, A. M., Al-Ani, A., Loo, C. K., Powell, T. Y., Martin, D. M., Breakspear, M., et al. (2017). Predicting tDCS treatment outcomes of patients with major depressive disorder using automated EEG classification. J. Affect. Disord. 208, 597-603. doi: 10.1016/j.jad.2016.10.021

Arns, M., Cerquera, A., Gutiérrez, R. M., Hasselman, F., and Freund, J. A. (2014). Non-linear EEG analyses predict non-response to rTMS treatment in major
EEG, in combination with the LORETA source estimation analysis, provides a promising tool for predicting the outcome of treatment intervention, including tDCS.

\section{DATA AVAILABILITY}

The datasets generated for this study can be found in Kansai Medical University, KAKEN 26860950 and KAKEN 19K08056.

\section{ETHICS STATEMENT}

The study protocol was approved by the Institutional Ethics Review Committee of Kansai Medical University.

\section{AUTHOR CONTRIBUTIONS}

KN and YM designed the study. KN, KK, SI, and MY recruited the participants. YK, SU, and RP-M analyzed the data. KN wrote the first draft. YM, RP-M, RI, and TK wrote the final manuscript.

\section{FUNDING}

This project was sponsored by a Grant-in-Aid for Young Scientists (B) (KAKEN 26860950) and Grant-in-Aid for Scientific Research (C) (KAKEN 19K08056) from the Japan Society for the Promotion of Science (JSPS), Translational Research Program: TR-SPRINT (Strategic Promotion for Practical Application of Innovative Medical Technology) from the Japan Agency for Medical Research and Development, AMED, and the Center of Innovation Program from Japan Science and Technology Agency, JST. The funders had no role in study design, data collection or analysis, the decision to publish, or preparation of the manuscript.

\section{ACKNOWLEDGMENTS}

We thank Benjamin Knight, MSc, from Edanz Group (www.edanzediting.com/ac) for editing a draft of this manuscript.

depressive disorder. Clin. Neurophysiol. 125, 1392-1399. doi: 10.1016/j.clinph. 2013.11.022

Bailey, N., Hoy, K., Rogasch, N., Thomson, R., Mcqueen, S., Elliot, D., et al. (2018). Responders to rTMS for depression show increased fronto-midline theta and theta connectivity compared to non-responders. Brain Stimul. 11, 190-203. doi: 10.1016/j.brs.2017.10.015

Bakker, N., Shahab, S., Giacobbe, P., Blumberger, D. M., Daskalakis, Z. J., Kennedy, S. H., et al. (2015). rTMS of the dorsomedial prefrontal cortex for major depression: safety, tolerability, effectiveness, and outcome predictors for 10 $\mathrm{Hz}$ versus intermittent theta-burst stimulation. Brain Stimul. 8, 208-215. doi: 10.1016/j.brs.2014.11.002

Brunoni, A. R., Moffa, A. H., Sampaio-Junior, B., Borrione, L., Moreno, M. L., Fernandes, R. A., et al. (2017). Trial of electrical direct-current therapy versus 
escitalopram for depression. N. Engl. J. Med. 376, 2523-2533. doi: 10.1056/ nejmoa1612999

Brunoni, A. R., Nitsche, M. A., Bolognini, N., Bikson, M., Wagner, T., Merabet, L., et al. (2012). Clinical research with transcranial direct current stimulation (tDCS): challenges and future directions. Brain Stimul. 5, 175-195. doi: 10. 1016/j.brs.2011.03.002

Brunoni, A. R., Valiengo, L., Baccaro, A., Zanao, T. A., De Oliveira, J. F., Goulart, A., et al. (2013). The sertraline vs. electrical current therapy for treating depression clinical study: results from a factorial, randomized, controlled trial. JAMA Psychiatry 70, 383-391. doi: 10.1001/2013.jamapsychiatry.32

Bush, G., Luu, P., and Posner, M. I. (2000). Cognitive and emotional influences in anterior cingulate cortex. Trends Cogn. Sci. 4, 215-222. doi: 10.1016/s13646613(00)01483-2

Colzato, L. S., Sellaro, R., Van Den Wildenberg, W. P., and Hommel, B. (2015). tDCS of medial prefrontal cortex does not enhance interpersonal trust. J. Psychophysiol. 29, 131-134. doi: 10.1027/0269-8803/a000144

Downar, J., and Daskalakis, Z. J. (2013). New targets for rTMS in depression: a review of convergent evidence. Brain Stimul. 6, 231-240. doi: 10.1016/j.brs. 2012.08.006

Downar, J., Geraci, J., Salomons, T. V., Dunlop, K., Wheeler, S., Mcandrews, M. P., et al. (2014). Anhedonia and reward-circuit connectivity distinguish nonresponders from responders to dorsomedial prefrontal repetitive transcranial magnetic stimulation in major depression. Biol. Psychiatry 76, 176-185. doi: 10.1016/j.biopsych.2013.10.026

Eldaief, M. C., Halko, M. A., Buckner, R. L., and Pascual-Leone, A. (2011). Transcranial magnetic stimulation modulates the brain's intrinsic activity in a frequency-dependent manner. Proc. Nat. Acad. Sci. 108, 21229-21234. doi: 10.1073/pnas.1113103109

Fox, M. D., Buckner, R. L., Liu, H., Chakravarty, M. M., Lozano, A. M., and Pascual-Leone, A. (2014). Resting-state networks link invasive and noninvasive brain stimulation across diverse psychiatric and neurological diseases. Proc. Nat. Acad. Sci. 111, E4367-E4375. doi: 10.1073/pnas.1405003111

Fregni, F., Nitsche, M., Loo, C., Brunoni, A., Marangolo, P., Leite, J., et al. (2015). Regulatory considerations for the clinical and research use of transcranial direct current stimulation (tDCS): review and recommendations from an expert panel. Clin. Res. Regul. Affairs 32, 22-35. doi: 10.3109/10601333.2015.980944

Fuchs, M., Kastner, J., Wagner, M., Hawes, S., and Ebersole, J. S. (2002). A standardized boundary element method volume conductor model. Clin. Neurophysiol. 113, 702-712. doi: 10.1016/s1388-2457(02)00030-5

Goldin, P. R., Manber-Ball, T., Werner, K., Heimberg, R., and Gross, J. J. (2009). Neural mechanisms of cognitive reappraisal of negative self-beliefs in social anxiety disorder. Biol. Psychiatry 66, 1091-1099. doi: 10.1016/j.biopsych.2009. 07.014

Goodkind, M., Eickhoff, S. B., Oathes, D. J., Jiang, Y., Chang, A., Jones-Hagata, L. B., et al. (2015). Identification of a common neurobiological substrate for mental illness. JAMA Psychiatry 72, 305-315. doi: 10.1001/jamapsychiatry.2014. 2206

Greenblatt, R. E., Ossadtchi, A., and Pflieger, M. E. (2005). Local linear estimators for the bioelectromagnetic inverse problem. IEEE Trans. Signal Process. 53, 3403-3412. doi: 10.1109/tsp.2005.853201

Hasler, G., Fromm, S., Alvarez, R. P., Luckenbaugh, D. A., Drevets, W. C., and Grillon, C. (2007). Cerebral blood flow in immediate and sustained anxiety. J. Neurosci. 27, 6313-6319. doi: 10.1523/jneurosci.5369-06.2007

Jacobson, L., Ezra, A., Berger, U., and Lavidor, M. (2012). Modulating oscillatory brain activity correlates of behavioral inhibition using transcranial direct current stimulation. Clin. Neurophysiol. 123, 979-984. doi: 10.1016/j.clinph. 2011.09.016

Kaiser, R. H., Andrews-Hanna, J. R., Wager, T. D., and Pizzagalli, D. A. (2015). Large-scale network dysfunction in major depressive disorder: a meta-analysis of resting-state functional connectivity. JAMA Psychiatry 72, 603-611. doi: 10. 1001/jamapsychiatry.2015.0071

Keeser, D., Padberg, F., Reisinger, E., Pogarell, O., Kirsch, V., Palm, U., et al. (2011). Prefrontal direct current stimulation modulates resting EEG and eventrelated potentials in healthy subjects: a standardized low resolution tomography (sLORETA) study. Neuroimage 55, 644-657. doi: 10.1016/j.neuroimage.2010. 12.004

Kitaura, Y., Nishida, K., Yoshimura, M., Mii, H., Katsura, K., Ueda, S., et al. (2017). Functional localization and effective connectivity of cortical theta and alpha oscillatory activity during an attention task. Clin. Neurophysiol. Pract. 2, 193-200. doi: 10.1016/j.cnp.2017.09.002

Klimesch, W. (1999). EEG alpha and theta oscillations reflect cognitive and memory performance: a review and analysis. Brain Res. Rev. 29, 169-195. doi: 10.1016/s0165-0173(98)00056-3

Kreuzer, P. M., Lehner, A., Schlee, W., Vielsmeier, V., Schecklmann, M., Poeppl, T. B., et al. (2015). Combined rTMS treatment targeting the anterior cingulate and the temporal cortex for the treatment of chronic tinnitus. Sci. Rep. 5:18028. doi: $10.1038 /$ srep 18028

Kupfer, D. J., Frank, E., and Phillips, M. L. (2012). Major depressive disorder: new clinical, neurobiological, and treatment perspectives. Lancet 379, 1045-1055. doi: 10.1016/s0140-6736(11)60602-8

Li, C.-T., Hsieh, J.-C., Huang, H.-H., Chen, M.-H., Juan, C.-H., Tu, P.-C., et al. (2014). Cognition-modulated frontal activity in prediction and augmentation of antidepressant efficacy: a randomized controlled pilot study. Cereb. Cortex 26, 202-210. doi: 10.1093/cercor/bhu191

Liston, C., Chen, A. C., Zebley, B. D., Drysdale, A. T., Gordon, R., Leuchter, B., et al. (2014). Default mode network mechanisms of transcranial magnetic stimulation in depression. Biol. Psychiatry 76, 517-526. doi: 10.1016/j.biopsych. 2014.01.023

Martin, D. M., Moffa, A., Nikolin, S., Bennabi, D., Brunoni, A. R., Flannery, W., et al. (2018). Cognitive effects of transcranial direct current stimulation treatment in patients with major depressive disorder: an individual patient data meta-analysis of randomised, sham-controlled trials. Neurosci. Biobehav. Rev. 90, 137-145. doi: 10.1016/j.neubiorev.2018.04.008

Mayberg, H. S., Lozano, A. M., Voon, V., Mcneely, H. E., Seminowicz, D., Hamani, C., et al. (2005). Deep brain stimulation for treatment-resistant depression. Neuron 45, 651-660.

Mazziotta, J., Toga, A., Evans, A., Fox, P., Lancaster, J., Zilles, K., et al. (2001). A probabilistic atlas and reference system for the human brain: international Consortium for Brain Mapping (ICBM). Philos. Trans. R. Soc. London B Biol. Sci. 356, 1293-1322. doi: 10.1098/rstb.2001.0915

McGrath, C. L., Kelley, M. E., Holtzheimer, P. E., Dunlop, B. W., Craighead, W. E., Franco, A. R., et al. (2013). Toward a neuroimaging treatment selection biomarker for major depressive disorder. JAMA Psychiatry 70, 821-829. doi: 10.1001/jamapsychiatry.2013.143

Mondino, M., Thiffault, F., and Fecteau, S. (2015). Does non-invasive brain stimulation applied over the dorsolateral prefrontal cortex non-specifically influence mood and emotional processing in healthy individuals? Front. Cell. Neurosci. 9:399. doi: 10.3389/fncel.2015.00399

Moore, J. P., Trudeau, D. L., Thuras, P. D., Rubin, Y., Stockley, H., and Dimond, T. (2000). Comparison of alpha-theta, alpha and EMG neurofeedback in the production of alpha-theta crossover and the occurrence of visualizations. J. Neurother. 4, 29-42. doi: 10.1300/j184v04n01_04

Murray, C. J., and Lopez, A. D. (1996). Evidence-based health policy--lessons from the Global Burden of Disease Study. Science 274, 740-743. doi: 10.1126/science. 274.5288.740

Mutz, J., Edgcumbe, D. R., Brunonimd, A. R., and Fumd, C. H. (2018). Efficacy and acceptability of non-invasive brain stimulation for the treatment of adult unipolar and bipolar depression: a systematic review and meta-analysis of randomised sham-controlled trials. Neurosci. Biobehav. Rev. 92, 291-303. doi: 10.1016/j.neubiorev.2018.05.015

Nishida, K., Razavi, N., Jann, K., Yoshimura, M., Dierks, T., Kinoshita, T., et al. (2015). Integrating different aspects of resting brain activity: a review of electroencephalographic signatures in resting state networks derived from functional magnetic resonance imaging. Neuropsychobiology 71, 6-16. doi: $10.1159 / 000363342$

Pascual-Marqui, R., Sekihara, K., Brandeis, D., and Michel, C. (2009). Imaging the Electrical Neuronal Generators of EEG/MEG. Cambridge, MA: Cambridge University Press.

Pascual-Marqui, R. D. (2002). Standardized low-resolution brain electromagnetic tomography (sLORETA): technical details. Methods Find Exp. Clin. Pharmacol. 24, 5-12.

Pascual-Marqui, R. D. (2007). Discrete, 3D distributed, linear imaging methods of electric neuronal activity. Part 1: exact, zero error localization. arXiv

Pizzagalli, D. A., Nitschke, J. B., Oakes, T. R., Hendrick, A. M., Horras, K. A., Larson, C. L., et al. (2002). Brain electrical tomography in depression: the 
importance of symptom severity, anxiety, and melancholic features. Biol. Psychiatry 52, 73-85. doi: 10.1016/s0006-3223(02)01313-6

Pizzagalli, D. A., Webb, C. A., Dillon, D. G., Tenke, C. E., Kayser, J., Goer, F., et al. (2018). Pretreatment rostral anterior cingulate cortex theta activity in relation to symptom improvement in depression: a randomized clinical trial. JAMA Psychiatry 75, 547-554. doi: 10.1001/jamapsychiatry.2018.0252

Schulze, L., Feffer, K., Lozano, C., Giacobbe, P., Daskalakis, Z. J., Blumberger, D. M., et al. (2018). Number of pulses or number of sessions? an open-label study of trajectories of improvement for once-vs. twice-daily dorsomedial prefrontal rTMS in major depression. Brain Stimul. 11, 327-336. doi: 10.1016/j.brs.2017. 11.002

Sekihara, K., Sahani, M., and Nagarajan, S. S. (2005). Localization bias and spatial resolution of adaptive and non-adaptive spatial filters for MEG source reconstruction. Neuroimage 25, 1056-1067. doi: 10.1016/j.neuroimage.2004.11. 051

Sha, Z., Wager, T. D., Mechelli, A., and He, Y. (2019). Common dysfunction of large-scale neurocognitive networks across psychiatric disorders. Biol. Psychiatry 85, 379-388. doi: 10.1016/j.biopsych.2018.11.011

Sheline, Y. I., Barch, D. M., Price, J. L., Rundle, M. M., Vaishnavi, S. N., Snyder, A. Z., et al. (2009). The default mode network and self-referential processes in depression. Proc. Nat. Acad. Sci. 106, 1942-1947. doi: 10.1073/pnas.081268 6106

Sylvester, C., Corbetta, M., Raichle, M., Rodebaugh, T., Schlaggar, B., Sheline, Y., et al. (2012). Functional network dysfunction in anxiety and anxiety disorders. Trends Neurosci. 35, 527-535. doi: 10.1016/j.tins.2012.04.012

Tremblay, S., Lepage, J. F., Latulipe-Loiselle, A., Fregni, F., Pascual-Leone, A., and Theoret, H. (2014). The uncertain outcome of prefrontal tDCS. Brain Stimul. 7, 773-783. doi: 10.1016/j.brs.2014.10.003
Ulam, F., Shelton, C., Richards, L., Davis, L., Hunter, B., Fregni, F., et al. (2015). Cumulative effects of transcranial direct current stimulation on EEG oscillations and attention/working memory during subacute neurorehabilitation of traumatic brain injury. Clin. Neurophysiol. 126, 486-496. doi: 10.1016/j.clinph.2014.05.015

van der Werf, Y. D., Sanz-Arigita, E. J., Menning, S., and Van Den Heuvel, O. A. (2010). Modulating spontaneous brain activity using repetitive transcranial magnetic stimulation. BMC Neurosci. 11:145. doi: 10.1186/1471-2202-11-145

Weigand, A., Horn, A., Caballero, R., Cooke, D., Stern, A. P., Taylor, S. F., et al. (2018). Prospective validation that subgenual connectivity predicts antidepressant efficacy of transcranial magnetic stimulation sites. Biol. Psychiatry 84, 28-37. doi: 10.1016/j.biopsych.2017.10.028

Wirth, M., Rahman, R. A., Kuenecke, J., Koenig, T., Horn, H., Sommer, W., et al. (2011). Effects of transcranial direct current stimulation (tDCS) on behaviour and electrophysiology of language production. Neuropsychologia 49, 3989-3998. doi: 10.1016/j.neuropsychologia.2011.10.015

Conflict of Interest Statement: The authors declare that the research was conducted in the absence of any commercial or financial relationships that could be construed as a potential conflict of interest.

Copyright (c) 2019 Nishida, Koshikawa, Morishima, Yoshimura, Katsura, Ueda, Ikeda, Ishii, Pascual-Marqui and Kinoshita. This is an open-access article distributed under the terms of the Creative Commons Attribution License (CC BY). The use, distribution or reproduction in other forums is permitted, provided the original author(s) and the copyright owner(s) are credited and that the original publication in this journal is cited, in accordance with accepted academic practice. No use, distribution or reproduction is permitted which does not comply with these terms. 